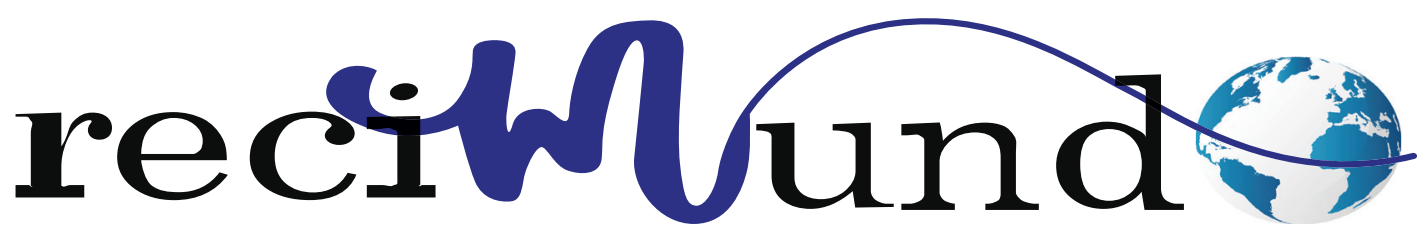

Revista Científica Mundo de la Investigación y el Conocimiento

DOI: 10.26820/recimundo/5.(Suple1).oct.2021.75-86

URL: https://recimundo.com/index.php/es/article/view/1334

EDITORIAL: Saberes del Conocimiento; Universidad Yachay Tech

REVISTA: RECIMUNDO

ISSN: 2588-073X

TIPO DE INVESTIGACIÓN: Artículo de revisión

CÓdigo UNESCO: 32 Ciencias Médicas

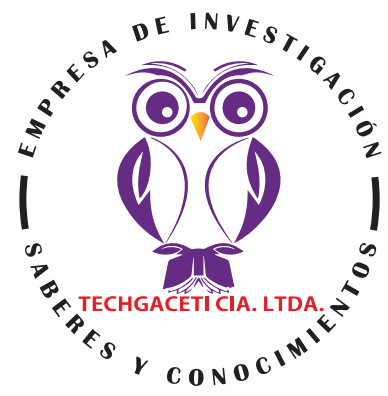

PAGINAS: 75-86

\title{
Ventajas del uso de anestesia epidural en cirugía abdominal
}

Advantages of the use of epidural anesthesia in abdominal surgery

Vantagens do uso da anestesia peridural em cirurgia abdominal

Hugo Valentín Saquipay Ortega'; Rubén Emmanuel Bravo Paucar²

RECIBIDO: 02/09/2021 ACEPTADO: 20/09/2021 PUBLICADO: 30/10/2021

1. Médico General; Universidad Católica de Cuenca; Médico Residente en la Clínica y Maternidad Alban Valarezo; Cuenca, Ecuador; drhugo_so134@hotmail.com; (D) https://orcid.org/0000-0002-3980-1484.

2. Medico General; Universidad Católica de Cuenca; Médico de Atención Primaria en el Centro de Salud Capitán Augusto Rivadeneyra; Orellana, Ecuador; rubenbravopaucar@gmail.com; (D) https://orcid.org/0000-00032108-5333

CORRESPONDENCIA

Rubén Emmanuel Bravo Paucar

rubenbravopaucar@gmail.com

Cuenca, Ecuador

() RECIMUNDO; Editorial Saberes del Conocimiento, 2021 


\section{RESUMEN}

Antecedentes: Desde 1980, la anestesia epidural se ha propuesto para controlar el dolor post- operatorio, consiste en administrar un anestésico local a nivel del espacio peridural con el fin de bloquear la transmisión del impulso doloroso. Objetivo: Describir las ventajas acerca del uso de anestesia epidural en cirugía abdominal. Fuente de datos: Se trata de una revisión bibliográfica durante los meses de julio - agosto 2020, en las bases de datos Google Scholar, PubMed y Elsevier obteniendo 352 trabajos, que tras la eliminación de resultados duplicados y análisis de calidad de evidencia generaron 30 estudios que se incluyeron como fuente de datos. Resultados: La anestesia epidural puede ser indicada en cirugía vascular abdominal, cirugía renal, cirugía ginecológica, y cirugía emergente en el contexto de COVID-19, además de todos aquellos pacientes con disminución previa de la función pulmonar que tengan contraindicaciones para anestesia general. El uso combinado con la anestesia general se asoció con importantes beneficios de la ventilación mecánica y una disminución de la estancia hospitalaria en pacientes críticos. Sin disminuir la inmunosupresión y pudiendo continuarse para aliviar el dolor posoperatorio. Limitaciones: La evidencia estudiada pertenece a poblaciones no latinas. El tipo de investigaciones incluidos para la revisión bibliográfica no fue homogéneo en su modalidad ni en el procedimiento quirúrgico en el cual se indicó. Conclusiones: La anestesia epidural por si sola presenta varias ventajas en múltiples procedimientos quirúrgicos abdominales, especialmente en aquellos realizados en pacientes con deterioro previo de la función pulmonar o alto riesgo de complicaciones, posee altos niveles de seguridad y viabilidad de utilización, sin embargo, su uso deberá estar basado en un protocolo que permita seleccionar los pacientes que se beneficiaran de ella.

Palabras clave: Anestesia Epidural, Espacio Epidural, Cirugía General.

\section{ABSTRACT}

Background: Since 1980, epidural anesthesia has been proposed to manage postoperative pain. It consists of a local anesthetic administered at the level of the peridural space in an attempt to block the transmission of the painful stimulus. Objective: To describe the advantages about the use of epidural anesthesia in abdominal surgery. Data source: This is a bibliographic review during the months of July - August 2020, in the Google Scholar, PubMed and Elsevier databases obtaining 352 works, that after eliminating duplicated results and quality evidence analysis generated 30 studies that have been included as a data source. Results: Epidural anesthesia can be indicated in abdominal vascular surgery, renal surgery, gynecological surgery, and emergent surgery in the context of COVID-19, in addition to all those patients with previous decrease in lung function and who have contraindications for general anesthesia. The combined use with general anesthesia was associated with significant benefits of mechanical ventilation and decreased hospital stay in critical patients. Without decreasing immunosuppression and can be continued to relieve postoperative pain. Limitations: The evidence studied belongs to non-Latino groups. The type of research involved in the literature review was not homogeneous in its modality or in the surgical procedure in which it was indicated. Conclusions: Epidural anesthesia alone has several advantages in multiple abdominal surgical procedures, especially those performed on patients with previous deterioration of lung function or high risk of complications. It has high levels of safety and feasibility of use, but should be based on a protocol that allows for the selection of patients who will benefit from it.

Keywords: Epidural Anesthesia, Epidural Space, General Surgery.

\section{RESUMO}

Introdução: Desde 1980, a anestesia peridural tem sido proposta para controlar a dor pós-operatória. Consiste em um anestésico local administrado ao nível do espaço peridural na tentativa de bloquear a transmissão do estímulo doloroso. Objetivo: Descrever as vantagens do uso da anestesia peridural em cirurgia abdominal. Fonte de dados: Esta é uma revisão bibliográfica durante os meses de julho a agosto de 2020, nas bases de dados Google Scholar, PubMed e Elsevier obtendo 352 trabalhos, que após eliminação de resultados duplicados e análise de evidências de qualidade gerou 30 estudos que foram incluídos como fonte de dados. Resultados: A anestesia peridural pode ser indicada em cirurgia vascular abdominal, cirurgia renal, cirurgia ginecológica e cirurgia de emergência no contexto do COVID-19, além de todas as pacientes com diminuição prévia da função pulmonar e que tenham contra-indicação para anestesia geral. O uso combinado com anestesia geral foi associado a benefícios significativos da ventilação mecânica e diminuição da permanência hospitalar em pacientes críticos. Sem diminuir a imunossupressão e pode ser continuado para aliviar a dor pós-operatória. Limitações: As evidências estudadas pertencem a grupos não latinos. O tipo de pesquisa envolvida na revisão da literatura não foi homogênea na modalidade e nem no procedimento cirúrgico em que foi indicada. Conclusões: A anestesia peridural isolada apresenta várias vantagens em vários procedimentos cirúrgicos abdominais, especialmente aqueles realizados em pacientes com deterioração prévia da função pulmonar ou alto risco de complicações. Apresenta elevados níveis de segurança e viabilidade de uso, mas deve ser baseado em um protocolo que permita a seleção dos pacientes que irão se beneficiar.

Keywords: Anestesia Epidural, Espaço Epidural, Cirurgia Geral. 


\section{Introducción}

Desde 1980, la anestesia epidural (AE) se ha propuesto para manejar el dolor postoperatorio, en especial en cirugías abdominales. Ésta es percibida por varios autores como la analgesia ideal para las cirugías abdominales mayores (gastrectomías y hepatectomías, entre otras) y además ha demostrado mejorar la morbimortalidad quirúrgica (1).

La técnica consiste en introducir un catéter a nivel del espacio peridural (entre duramadre y ligamento amarillo), se administra un anestésico local y/u opioide produciendo analgesia y bloqueo simpático. Para cirugías abdominales mayores se debe alcanzar un nivel mínimo de T6, dermatoma que corresponde al apéndice xifoides (2).

La primera causa de muerte durante el postoperatorio de una cirugía mayor, es la cardíaca. La excesiva activación de T1-T5 a causa del estrés quirúrgico, aumenta la demanda de oxígeno miocárdico y vasoconstricción coronaria, además de desencadenar un estado de hipercoagulabilidad.

La anestesia espinal torácica, al producir un bloqueo selectivo de la inervación simpática cardíaca (T1-T5), produce disminución del efecto adrenérgico, reduciendo la presión arterial, frecuencia y contractibilidad cardíaca, que son los mayores determinantes de la demanda de oxígeno miocárdico durante la intervención quirúrgica. Adicionalmente, el bloqueo produce vasodilatación coronaria y sistémica, aumentando el flujo sanguíneo en las regiones isquémicas del miocardio, y también una reducción en la precarga y poscarga $(3,4)$.

Los eventos tromboembólicos en el período postoperatorio se asocian a un estado de hipercoagulabilidad producido por el acto quirúrgico. Habrá una reducción en el flujo venoso dado por la ventilación mecánica (presión positiva), el bloqueo neuromuscu- lar y la activación del sistema simpático. Este último aumenta el factor VIII y de Von Willebrand, inhibe la fibrinólisis a través del PAI-1, disminuye la antitrombina III e inicia la agregación plaquetaria. La AE, al producir bloqueo simpático, disminuye los efectos de este sobre la coagulación, reduciendo el riesgo de tromboembólico (5).

El impacto de la AE sobre la función pulmonar es ambiguo, sin embargo, puede disminuir la instrumentalización de la vía aérea, la necesidad de ventilación mecánica, incidencia de atelectasia e infecciones pulmonares y la estancia hospitalaria. En el postoperatorio inmediato, la función pulmonar dependerá de la relajación muscular residual, la disfunción diafragmática, el momento de extubación, la disminución de la complacencia pulmonar y el dolor. La AE bloquea el arco reflejo del nervio frénico mediante el anestésico local, permitiendo una mejor función diafragmática $(4,6)$.

La anestesia espinal torácica, al producir un bloqueo selectivo de la inervación simpática cardíaca (T1-T5), produce disminución del efecto adrenérgico, reduciendo la presión arterial, frecuencia y contractibilidad cardíaca, que son los mayores determinantes de la demanda de oxígeno miocárdico durante la intervención quirúrgica. Adicionalmente, el bloqueo produce vasodilatación coronaria y sistémica, aumentando el flujo sanguíneo en las regiones isquémicas del miocardio, y también una reducción en la precarga y poscarga $(3,4)$.

Los eventos tromboembólicos en el período postoperatorio se asocian a un estado de hipercoagulabilidad producido por el acto quirúrgico. Habrá una reducción en el flujo venoso dado por la ventilación mecánica (presión positiva), el bloqueo neuromuscular y la activación del sistema simpático. Este último aumenta el factor VIII y de Von Willebrand, inhibe la fibrinólisis a través del PAI-1, disminuye la antitrombina III e inicia la agregación plaquetaria. La AE, al produ-

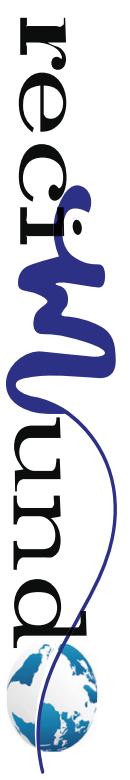


cir bloqueo simpático, disminuye los efectos de éste sobre la coagulación, reduciendo el riesgo de tromboembólico (5).

El impacto de la AE sobre la función pulmonar es ambiguo, sin embargo, puede disminuir la instrumentalización de la vía aérea, la necesidad de ventilación mecánica, incidencia de atelectasia e infecciones pulmonares y la estancia hospitalaria. En el postoperatorio inmediato, la función pulmonar dependerá de la relajación muscular residual, la disfunción diafragmática, el momento de extubación, la disminución de la complacencia pulmonar y el dolor. La AE bloquea el arco reflejo del nervio frénico mediante el anestésico local, permitiendo una mejor función diafragmática $(4,6)$.

\section{Metodología}

Se trata de un estudio de revisión bibliográfica, elaborado en base a la revisión de literatura internacional de los últimos 5 años el cual se realizó durante los meses de Julio - Agosto, en las bases de datos Google Scholar, PubMed y Elsevier incluyendo publicaciones en español e inglés, en humanos adultos de 18 a 65 años de edad, durante los últimos cinco años, con las palabras "Anesthesia, Epidural" AND "abdominal surgery".

\section{Criterios de Inclusión:}

Textos en inglés y en español, publicados desde el año 2015, con metodología basada en evidencia y alojados en las bases de datos mencionadas.

\section{Criterios de Exclusión:}

Textos duplicados, que hayan declarado conflictos de intereses y aquellos que no posean declaración explicita de no presentar conflictos de intereses.

Se encontraron 352 trabajos (Google Scholar 204, PubMed 37 y Elsevier 111), tras descartar los trabajos repetidos (32 trabajos), y la revisión de títulos y resúmenes se se- leccionaron 56 estudios que fueron validados mediante listas guía de comprobación de artículos científicos (PRISMA, STROBE, CONSORT, etc.) según el tipo de estudio, quedando 30 estudios incluidos en esta revisión.

Todos los datos fueron manejados por los autores en absoluta confidencialidad y siguiendo estamentos internacionales para la publicación biomédica. 
Gráfico 1. Algoritmo de selección de pacientes

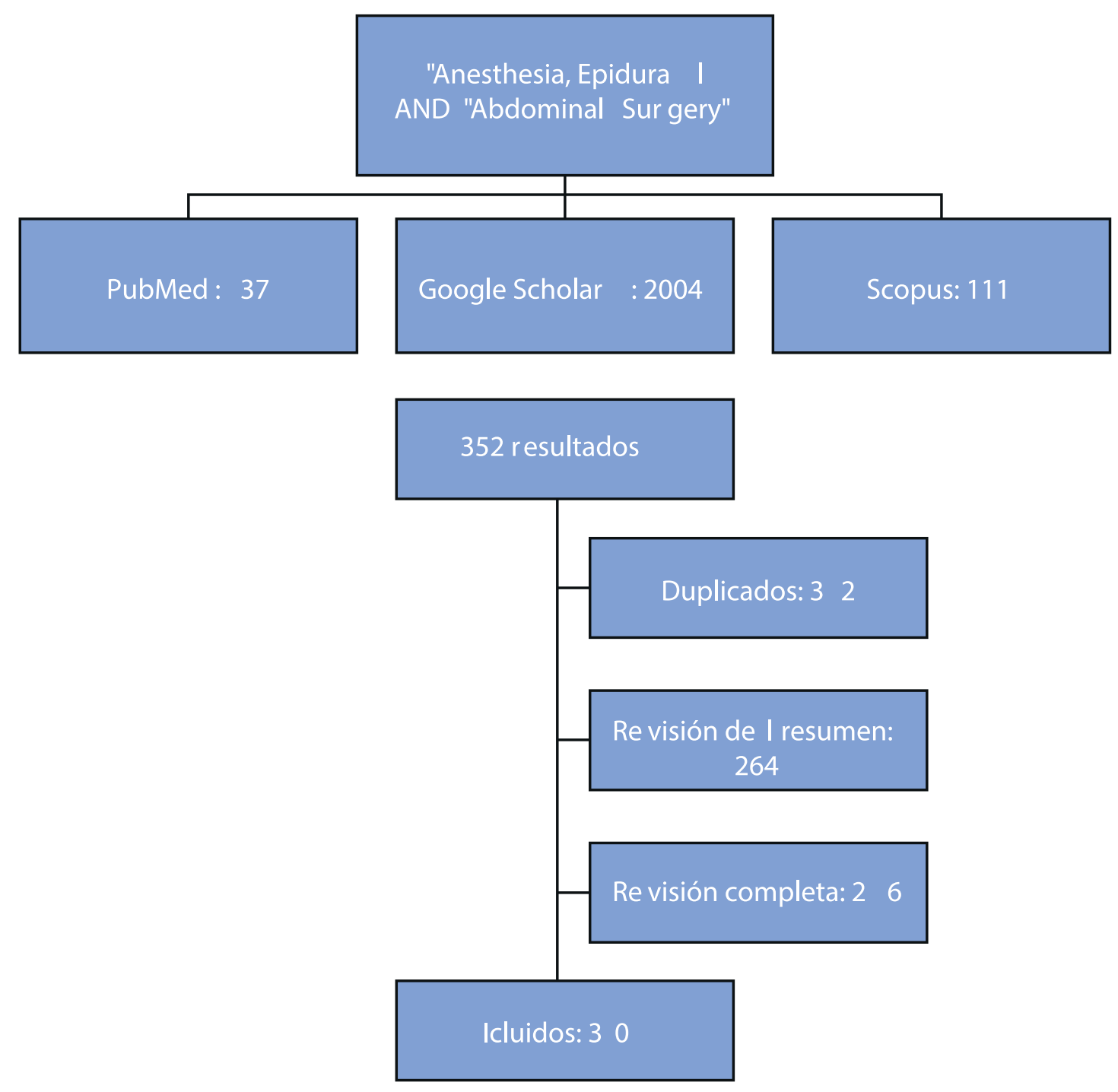

Elaboración: Los autores.

\section{Calidad de estudios y sesgo.}

En esta revisión se incluyeron cinco revisiones sistemáticas (7-11); once estudios de cohorte retrospectiva $(1,12-21)$; tres de cohorte prospectiva $(3,22,23)$; siete ensayos clínicos controlados aleatorizados (2430); dos ensayos clínicos no aleatorizado $(31,32)$; un reporte de caso (33); y un metaanálisis de ensayos clínicos controlados aleatorizados (34).

La calidad de la evidencia se midió utilizando las listas de verificación mencionadas en el apartado de metodología; a lo que la mayoría de artículos cumplieron con los requisitos propuestos según cada tipo de estudio. Ningún artículo reportó conflicto de interés.

\section{Resultados}

\section{Síntesis de resultados}

\section{Indicaciones para el uso de anestesia epidural}

La anestesia epidural es una medida aceptada de control del dolor después de una cirugía abdominal mayor. Sin embargo, si la anestesia epidural no tiene éxito, pueden

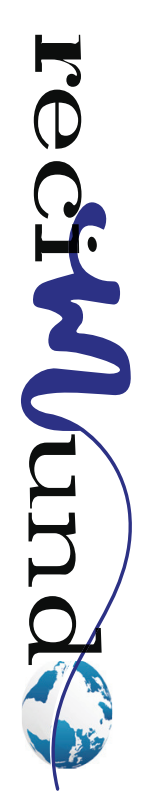


ocurrir diversos efectos adversos: respuesta al estrés excesivo, escasa movilización del paciente, aumento del uso de opioides e hipotensión debido a la vasodilatación (13). ¿En qué procedimientos se prefiere el uso de anestesia epidural?

- Reparación de aneurisma aórtico abdominal (12)

- Feocromocitoma (14)

- Cesárea (25)

- Cirugía abdominal de emergencia en contexto de COVID-19 (23)

\section{Ventajas de anestesia epidural}

Los bloqueos epidurales tienen efectos beneficiosos sobre los órganos vitales durante la cirugía abdominal. Sin embargo, un estudio fue llevado a cabo, al existir poca evidencia disponible acerca de la influencia de este tipo de anestesia sobre la permeabilidad intestinal durante cirugía aórtica abierta; concluyendo que, durante esta, el pinzamiento aórtico provocaba cambios desfavorables en la permeabilidad intestinal y que el bloqueo epidural no logró atenuar este deterioro (26).

Por otro lado, en un estudio llevado a cabo en pacientes sometidas a cesárea electiva que comparó bloqueo del plano transverso del abdomen (TAP) versus bloqueo epidural (EPI), se observó un mayor nivel de satisfacción del paciente en el grupo de bloqueo epidural ( $p=0.003$ ); la cantidad (mg) de analgésicos totales recibidos por las pacientes en las primeras 24 horas posoperatorias fue estadísticamente significativamente mayor ( $p=0.021$ ) en el grupo TAP en comparación con el grupo EPI; las puntuaciones de la escala visual análoga del dolor (EVA) del grupo EPI fueron significativamente más bajas en comparación con las del grupo TAP ( $p<0.001$ ). Concluyendo que, la anestesia epidural es el estándar de oro para lograr una adecuada analgesia post cesárea, proporcionando un control eficaz del dolor posoperatorio (25).
Otros estudios también confirman la efectividad de la anestesia epidural (incluso a dosis bajas) para cesárea, sin presentar eventos adversos como hipotensión, prurito, náusea, vómito, entre otros $(10,34)$.

Así mismo, la anestesia epidural demostró ser útil para pacientes embarazadas con enfermedad cardiovascular, logrando que la presión arterial sistólica no aumentara durante el trabajo de parto $(110.5 \mathrm{mmHg}$, IC 95\%; 95-132 mmHg frente a $110 \mathrm{mmHg}$, IC del 95\%; 91-130 mmHg), y reduciendo eventos cardiovasculares relacionados con la arritmia (16). Incluso, el uso de anestesia epidural se considera seguro para parto por cesárea en parturientas con COVID-19, con mortalidad nula y sin riesgo de morbilidad neonatal (18).

Una revisión sistemática encontró que en cirugía abdominal, una epidural que contiene un anestésico local, con o sin la adición de un opioide, acelera el regreso del tránsito gastrointestinal (diferencia media DME -0.67; IC 95\%: -0.86 a -0.47; equivalente a 22 horas) y puede reducir la estancia hospitalaria (DME -0.20; IC 95\%: -0.35 a -0.04; equivalente a un día); además que, una epidural que contiene un anestésico local con un opioide reduce el dolor al moverse 24 horas posquirúrgico (DME 0.89; IC 95\%: -1.08 a -0.70; equivalente a 2.5 en una escala de 0 a 10); sin embargo, no existió diferencia en la incidencia de vómitos dentro de las 24 horas (RR 0.84; IC 95\%: 0.57 a 1.23) o fuga anastomótica (RR 0.74; IC 95\%: 0.41 a 1.32) (8).

También se ha demostrado que la aplicación de anestesia epidural no provoca una reducción clínicamente significativa de la saturación cerebral durante la anestesia general en el curso de procedimientos quirúrgicos abdominales mayores (32).

Un interesante estudio reportó que en pacientes que necesitaron cirugía abdominal de emergencia durante la pandemia por 
COVID-19, el uso de anestesia loco regional, incluida anestesia epidural, permitió la ejecución segura de cirugía abierta con los pacientes despiertos; representando una alternativa útil en la era COVID-19 para pacientes frágiles que no pueden esperar. Además, ninguno de los pacientes fue intubado; el tiempo quirúrgico medio fue de 80 minutos (mínimo 30 minutos, máximo 130 minutos); el dolor intraoperatorio y posoperatorio fueron bien controlados; ningún paciente requirió cuidados intensivos posoperatorios y no se observaron complicaciones perioperatorias (23).

En un ensayo clínico aleatorizado realizado en pacientes con pancreatitis aguda se observó una mejora en la perfusión del páncreas en $43 \%$ de las mediciones donde se intervino con anestesia epidural $(p=$ 0.0025); además, la necesidad de necrosectomía fue menor $(p=0,63)$ y el dolor evaluado mediante EVA mejoró durante los primeros diez días $(p=0.034)$. Concluyendo que la anestesia epidural aumenta la perfusión arterial del páncreas y mejora el resultado clínico de los pacientes con pancreatitis aguda (27).

Un estudio retrospectivo llevado a cabo con pacientes que padecían carcinoma epitelial de ovario, trompas de Falopio o peritoneal en estadio IIIB-IV, que se sometieron a cirugía cito reductora primaria, reportó una mediana de supervivencia libre de progresión de 20.8 meses vs. 13.9 meses ( $p=0.021$ ), con el uso de anestesia epidural y no epidural, respectivamente; y una mediana de supervivencia general de 62.4 meses y 41.9 meses, respectivamente $(p<0.001)$. En este estudio el uso de anestesia epidural se asoció de forma independiente con un menor riesgo de progresión $(H R=1.327 ; \mathrm{IC} 95 \%$ $1.066-1.653)$ y muerte $(H R=1.588 ;$ IC $95 \%$ 1.224-2.06) (15).

Un reporte de caso menciona la realización de una gastrostomía laparoscópica con anestesia epidural, con el paciente despierto; aunque la anestesia general es de elección, dado que el paciente padecía enfermedad pulmonar obstructiva crónica y cáncer de pulmón estadio IV, el bloqueo neuromuscular inducido durante esta podía aumentar la atelectasia y la dependencia posoperatoria del ventilador, por lo que se prefirió la epidural, proporcionando suficiente anestesia y mejor cuidado posoperatorio para la gastrostomía laparoscópica (33).

En pacientes sometidos a resección hepática abierta con uso de anestesia epidural, un estudio retrospectivo reportó que el 89\% logró control del dolor posquirúrgico en reposo (escala de frecuencia numérica $<3$ ) y apenas el $8.2 \%$ tuvo dolor intenso con el movimiento (escala de frecuencia numérica> 6) (1).

También se ha evaluado el uso de anestesia epidural en cirugía retrógrada infrarrenal, demostrando que produce resultados favorables durante el periodo posoperatorio, con una puntuación media según EVA en el primer día posoperatorio de $0.82 \pm 1.3$, comparada con $1.20 \pm 0.9$ para anestesia general; considerándola una alternativa costo-efectiva a esta última (22).

Un ensayo clínico controlado aleatorizado pudo demostrar que el uso de anestesia epidural para colecistectomía laparoscópica preserva la estabilidad hemodinámica y proporciona mayor control del dolor al reducir los niveles de citocinas inflamatorias durante el período intraoperatorio y las primeras 24 horas del posoperatorio (28).

Otro estudio determinó que la cirugía asistida por laparoscopía para tumores anexales mediante una única incisión suprapúbica bajo anestesia epidural es factible y segura, y debe considerarse como un enfoque alternativo a la cirugía mínimamente invasiva convencional (31). 
Para pacientes sometidos a gastrectomía distal laparoscópica, con el uso de anestesia epidural el primer día de flatos fue significativamente más temprano (2.21 vs 2.44 días, $p=0.045$ ) y el número de dosis adicionales de analgésicos fue significativamente menor (2.85 vs 4.86 dosis, $p=0.007$ ) comparado con el no uso de la misma (21). La anestesia epidural ha demostrado ser efectiva en cuanto al manejo del dolor posquirúrgico en pacientes sometidos a este tipo de cirugía (3).

Una revisión sistemática demostró que el bloqueo neuro axial, incluida la anestesia epidural, manejado con cuidado, podría convertirse en una alternativa útil a la anestesia general en pacientes con estenosis aórtica; no ha demostrado alterar la hemodinamia (11).

Incluso la combinación de anestesia epidural/general muestra mejores resultados que la anestesia general sola: En análisis ajustados para reparación de aneurisma aórtico abdominal, el uso de anestesia combinada epidural-general se asoció con riesgos de mortalidad significativamente menores en comparación con el uso de anestesia general sola (índice de riesgo, 0.73; IC 95\% 0.57-0.92; $p=0.01$ ).

Los pacientes que recibieron anestesia combinada también tuvieron menores probabilidades de reintervención quirúrgica a los 30 días (odds ratio [OR], 0.65; IC 95\% $0.44-0.94 ; p=0.02$ ), así como isquemia intestinal posoperatoria (OR 0.54; IC 95\% 0.31-0.94; $p=0.03)$, complicaciones pulmonares (OR 0.62; IC 95\% 0.41-0.95; $p=$ $0.03)$ y necesidades de diálisis (OR, 0,44; IC 95\% 0,23-0,88; $P=.02$ ). No se observaron diferencias significativas para las probabilidades de complicaciones de la herida (OR 0.88; IC 95\%, 0.38-1.44; p = 0.51) y cardíacas (OR 1.08; IC 95\% 0.59-1.78; p $=0.82)(12)$.
De manera similar, un estudio retrospectivo con respecto a reparación de aneurisma aórtico abdominal, reportó que la anestesia combinada se asoció con riesgos significativamente menores de mortalidad por todas las causas (índice de riesgo $[\mathrm{HR}]=0,47$; intervalo de confianza [IC] 95\% 0.37-0.61) y eventos cardiovasculares adversos mayores $(\mathrm{HR}=0.72$; IC del 95\% 0.60- 0.86). Los pacientes combinados tenían probabilidades más bajas de lesión renal aguda (razón de probabilidades [OR] = 0.66; IC 95\% 0.49-0.89), insuficiencia respiratoria (OR = 0.41 ; IC 95\% 0.36-0.47) y complicaciones de las extremidades (OR $=0.30 ;$ IC 95\% 0.25-0.37), con mayores probabilidades de ser dado de alta (OR = 1.32; IC 95\% 1.151.51).

La anestesia combinada también se asoció con importantes beneficios de la ventilación mecánica y la UCl y la duración de la estancia hospitalaria. La anestesia combinada general y neuro axial en la reparación electiva de un aneurisma aórtico abdominal abierto se asocia con una reducción de la mortalidad y morbilidad a los 90 días. La anestesia neuro axial debe considerarse como un complemento de rutina de la anestesia general para la reparación electiva de aneurisma aórtico abdominal abierto (20).

Para pacientes sometidos a cirugía abierta por feocromocitoma, el uso de anestesia epidural general combinada disminuyó la incidencia de complicaciones postoperatorias ( $p=0.006)$. El análisis de regresión logística multivariante mostró que el uso de anestesia epidural-general combinada (OR 0.219; IC 95\%: 0.065-0.741; $p=0.015$ ) se asoció con un riesgo menor. (14).

El uso combinado de anestesia general con anestesia epidural logró niveles significativamente más bajos de TNF- $\alpha$, IL-6, IL-10, ACTH y citocinas inflamatorias (todos, $p$ $<0.05$ ), jugando un papel importante en la reducción de la respuesta inmune y al estrés en pacientes con cáncer de colon, des- 
de la cirugía hasta 24 horas después de la cirugía. (17).

En pacientes con cáncer de vesícula biliar el uso de anestesia epidural combinada con general podría atenuar las respuestas hemodinámicas intraoperatorias y mejorar la inmunidad celular posoperatoria (29).

Una combinación de anestesia general y epidural perioperatoria puede disminuir la inmunosupresión y aliviar el dolor posoperatorio y las náuseas o vómito posquirúrgicas en pacientes sometidos a resección de cáncer gástrico (30).

Un ensayo clínico controlado aleatorizado mostró que la anestesia epidural combinada puede mejorar la calidad y eficiencia de la laparoscopía para neoplasias de ovario, con las ventajas de una dosis reducida de anestésicos, analgesia posoperatoria satisfactoria, estabilidad hemodinámica mantenida, excelente relajación uterina y tiempo reducido de inducción de la anestesia, cirugía, recuperación y extubación (24).

En pacientes con cáncer ovárico sometidas a ooforectomía con anestesia epidural combinada con general se observaron menores niveles de expresión de CA-125 que en aquellas sometidas a anestesia general sola; la tasa de supervivencia a 5 años también aumentó significativamente en las primeras, determinando una mayor utilidad de anestesia epidural combinada comparada con anestesia general sola en este grupo de pacientes (19).

\section{Dlscusión}

La anestesia combinada se asocia con una mejor supervivencia y Hazard y odds ratio significativamente más bajos para la mortalidad y la morbilidad en pacientes sometidos a reparación electiva de aneurisma aórtico abdominal. El beneficio de supervivencia puede atribuirse a la reducción de los eventos adversos posoperatorios inmediatos (12).
Sin embargo, en procedimientos como pancreatectomía, se ha reportado que la anestesia epidural puede resultar disfuncional hasta en un 49\%; esto se asocia de forma independiente con el desarrollo de complicaciones generales ( $p<0.001$ ), relacionadas con el páncreas $(p=0.041)$ y no relacionadas con el páncreas $(p=0.001)$; y significa que los resultados post pancreatectomía pueden mejorarse aumentando la tasa de éxito de la anestesia epidural (13).

También cuando se utilizó en colecistectomías laparoscópicas, generó hipotensión intraoperatoria (RR 4.61 IC 95\%: 1.70-12.48, $\mathrm{p}=0.003$ ), bradicardia (RR 6,67 IC 95\%: 2.02-21.96, $p=0.002$ ), y cefalea posquirúrgica, con mayor frecuencia; además de asociarse a dolor intraoperatorio referido al hombro, requirió conversión anestésica en el $3.4 \%$ de los casos y no demostró evidencia de beneficios respiratorios para pacientes con función pulmonar normal (9).

Adicionalmente, la anestesia epidural puede aumentar la retención urinaria en comparación con no utilizarla (21).

Existen otras modalidades de anestesia epidural, por ejemplo, la torácica, que ha demostrado permitir movilización temprana, lograr funciones pulmonares posoperatorias satisfactorias con mayor rapidez, disminuir la incidencia de tos ineficaz, atelectasia e infecciones pulmonares; mejorar el flujo sanguíneo intestinal, prevenir reducciones en la perfusión del conducto gástrico y reducir la duración del íleo, en cirugías torácicas, sobre todo esofágicas.

Esta técnica permite una recuperación temprana de la anestesia, control eficaz del dolor posoperatorio y una duración significativamente más corta de la ventilación mecánica postoperatoria; aunque requiere habilidades técnicas específicas para una conducción óptima y no está exenta de riesgos, complicaciones y fracasos (7). 


\section{Limitaciones}

Esta investigación acerca del uso de anestesia epidural está limitada a que la evidencia incluida estudia poblaciones no latinoamericanas. También se considera limitaciones a la calidad de este estudio que el tipo de investigaciones incluidos para la revisión bibliográfica no fue homogéneo en su modalidad ni en el procedimiento quirúrgico en el cual se indicó, sin embargo, nos permiten tener una visión general de los beneficios que podría tener el usar la anestesia epidural.

\section{Conclusiones}

La anestesia epidural por si sola presenta varias ventajas en múltiples procedimientos quirúrgicos abdominales, especialmente en aquellos realizados en pacientes con deterioro previo de la función pulmonar o alto riesgo de complicaciones pulmonares, posee altos niveles de seguridad y viabilidad de utilización.

Entre sus aplicaciones, al bloquear la transmisión del impulso doloroso puede disminuir la generación del estrés inducido por la cirugía y la respuesta inflamatoria central, es así, que acompañada de una sedación consciente además de proporcionar una ventana óptima para la realización del procedimiento quirúrgico permite reducir la tasa complicaciones y disminuir el uso de analgesia en el postoperatorio.

Implicaciones para la práctica clínica:

Las ventajas reportadas de la aplicación de la anestesia epidural muestra enormes beneficios, sin embargo, debemos menciona que el estándar dorado para cirugía abdominal es la anestesia general, dejando la anestesia epidural sola para pacientes seleccionados de manera específica, o para complementar un protocolo de analgesia post operatoria multimodal.
Implicaciones para la investigación:

El uso de la anestesia epidural en el Ecuador es poco prevalente, consecuentemente se ha despreocupado la investigación sobre los beneficios de su aplicación. Este trabajo insta a la investigación acerca del uso de analgesia epidural en base al sistema de salud y la población del Ecuador para generar protocolos que permitan su aplicación cuando el paciente así lo amerite.

\section{Financiamiento}

Este proyecto ha sido financiado en su totalidad por los autores.

\section{Bibliografía}

1. Esteve N, Ferrer A, Sansaloni C, Mariscal M, Torres M, Mora C. Epidural anesthesia and analgesia in liver resection: Safety and effectiveness. Rev Esp Anestesiol Reanim. febrero de 2017;64(2):86-94.

2. Jing C, Wang C. Combining Spinal-Epidural Anesthesia versus Single-Shot Spinal Anesthesia for Cesarean Delivery: A Meta-Analysis of 5 Randomized Controlled Trials. Med Sci Monit. 18 de abril de 2019;25:2859-67.

3. Shibasaki S, Kawamura H, Homma S, Yosida T, Takahashi S, Takahashi M, et al. A comparison between fentanyl plus celecoxib therapy and epidural anesthesia for postoperative pain management following laparoscopic gastrectomy. Surg Today. octubre de 2016;46(10):1209-16.

4. Wink J, Veering BT, Aarts LPHJ, Wouters PF. Effects of Thoracic Epidural Anesthesia on Neuronal Cardiac Regulation and Cardiac Function. Anesthesiology. 1 de marzo de 2019;130(3):472-91.

5. Çakici MÇ, Özok HU, Erol D, Çatalca S, Sari S, Özdemir H, et al. Comparison of general anesthesia and combined spinal-epidural anesthesia for retrograde intrarenal surgery. Minerva Urol Nefrol. diciembre de 2019;71(6):636-43.

6. Avila-Hernandez AN, Singh P. Epidural Anesthesia. En: StatPearls [Internet]. Treasure Island (FL): StatPearls Publishing; 2020 [citado 4 de octubre de 2020]. Disponible en: http://www.ncbi.nlm.nih. gov/books/NBK542219/

7. Feltracco P, Bortolato A, Barbieri S, Michieletto E, Serra E, Ruol A, et al. Perioperative benefit and outcome of thoracic epidural in esophageal sur- 
gery: a clinical review. Dis Esophagus. 1 de mayo de 2018;31(5).

8. Guay J, Nishimori M, Kopp S. Epidural local anaesthetics versus opioid-based analgesic regimens for postoperative gastrointestinal paralysis, vomiting and pain after abdominal surgery. Cochrane Database Syst Rev. 16 de julio de 2016;7:CD001893.

9. Longo MA, Cavalheiro BT, de Oliveira Filho GR. Laparoscopic cholecystectomy under neuraxial anesthesia compared with general anesthesia: Systematic review and metaanalyses. J Clin Anesth. septiembre de 2017;41:48-54.

10. Van de Velde M. Low-dose spinal anesthesia for cesarean section to prevent spinal-induced hypotension. Curr Opin Anaesthesiol. junio de 2019;32(3):268-70.

11. Johansson S, Lind MN. Central regional anaesthesia in patients with aortic stenosis - a systematic review. Dan Med J. septiembre de 2017;64(9).

12. Bardia A, Sood A, Mahmood F, Orhurhu V, MueIler A, Montealegre-Gallegos $\mathrm{M}$, et al. Combined Epidural-General Anesthesia vs General Anesthesia Alone for Elective Abdominal Aortic Aneurysm Repair. JAMA Surg. 01 de 2016;151(12):1116-23.

13. Sugimoto M, Nesbit L, Barton JG, Traverso LW. Epidural anesthesia dysfunction is associated with postoperative complications after pancreatectomy. J Hepatobiliary Pancreat Sci. febrero de 2016;23(2):102-9.

14. Li N, Kong H, Li S-L, Zhu S-N, Wang D-X. Combined epidural-general anesthesia was associated with lower risk of postoperative complications in patients undergoing open abdominal surgery for pheochromocytoma: A retrospective cohort study. PLOS ONE. 2018;13(2):0192924.

15. Tseng JH, Cowan RA, Afonso AM, Zhou Q, lasonos A, Ali N, et al. Perioperative epidural use and survival outcomes in patients undergoing primary debulking surgery for advanced ovarian cancer. Gynecol Oncol. 2018;151(2):287-93.

16. Tanaka H, Kamiya C, Katsuragi S, Tanaka K, Yoshimatsu J, Ikeda T. Effect of epidural anesthesia in labor; pregnancy with cardiovascular disease. Taiwan J Obstet Gynecol. abril de 2018;57(2):190-3.

17. Hou B-J, Du Y, Gu S-X, Fan J, Wang R, Deng $\mathrm{H}$, et al. General anesthesia combined with epidural anesthesia maintaining appropriate anesthesia depth may protect excessive production of inflammatory cytokines and stress hormones in colon cancer patients during and after surgery. Medicine (Baltimore). julio de 2019;98(30):16610.
18. Chen R, Zhang Y, Huang L, Cheng B-H, Xia Z-Y, Meng Q-T. Safety and efficacy of different anesthetic regimens for parturients with COVID-19 undergoing Cesarean delivery: a case series of 17 patients. Can J Anaesth. 2020;67(6):655-63.

19. Han X-R, Wen X, Li Y-Y, Fan S-H, Zhang Z-F, Li $\mathrm{H}$, et al. Effect of different anesthetic methods on cellular immune functioning and the prognosis of patients with ovarian cancer undergoing oophorectomy. Biosci Rep. 31 de octubre de 2017;37(5).

20. Salata K, Abdallah FW, Hussain MA, de Mestral C, Greco E, Aljabri B, et al. Short-term outcomes of combined neuraxial and general anaesthesia versus general anaesthesia alone for elective open abdominal aortic aneurysm repair: retrospective population-based cohort study†. Br J Anaesth. 2020;124(5):544-52.

21. Yanagimoto Y, Takiguchi S, Miyazaki Y, Mikami J, Makino T, Takahashi T, et al. Comparison of pain management after laparoscopic distal gastrectomy with and without epidural analgesia. Surg Today. febrero de 2016;46(2):229-34.

22. Çakici MÇ, Özok HU, Erol D, Çatalca S, Sari S, Özdemir H, et al. Comparison of general anesthesia and combined spinal-epidural anesthesia for retrograde intrarenal surgery. Minerva Urol Nefrol. diciembre de 2019;71(6):636-43.

23. Romanzi A, Galletti M, Macchi L, Putortì A, Rossi F, Scolaro R, et al. Awake laparotomy: is locoregional anesthesia a functional option for major abdominal surgeries in the COVID19 era? Eur Rev Med Pharmacol Sci. 2020;24(9):5162-6.

24. Xu Q, Zhang H, Zhu Y-M, Shi N-J. Effects of Combined General/Epidural Anesthesia on Hemodynamics, Respiratory Function, and Stress Hormone Levels in Patients with Ovarian Neoplasm Undergoing Laparoscopy. Med Sci Monit. 8 de noviembre de 2016;22:4238-46.

25. Canakci E, Gultekin A, Cebeci Z, Hanedan B, Kilinc A. The Analgesic Efficacy of Transverse Abdominis Plane Block versus Epidural Block after Caesarean Delivery: Which One Is Effective? TAP Block? Epidural Block? Pain Res Manag. 2018;2018:3562701.

26. Owczuk R, Dylczyk-Sommer A, Wojciechowski J, Paszkiewicz M, Wujtewicz M, Stepnowski P, et al. The influence of epidural blockade on gut permeability in patients undergoing open surgical repair of abdominal aortic aneurysm. Anaesthesiol Intensive Ther. 2016;48(2):122-7.

27. Sadowski SM, Andres A, Morel P, Schiffer E, Frossard J-L, Platon A, et al. Epidural anesthesia

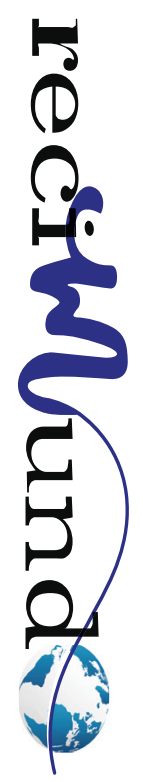


improves pancreatic perfusion and decreases the severity of acute pancreatitis. World J Gastroenterol. 21 de noviembre de 2015;21(43):12448-56.

28. Ozcan S, Ozer AB, Yasar MA, Erhan OL. Effects of combined general anesthesia and thoracic epidural analgesia on cytokine response in patients undergoing laparoscopic cholecystectomy. Niger J Clin Pract. agosto de 2016;19(4):436-42.

29. Zhu J, Zhang X-R, Yang $H$. Effects of combined epidural and general anesthesia on intraoperative hemodynamic responses, postoperative cellular immunity, and prognosis in patients with gallbladder cancer: A randomized controlled trial. Medicine (Baltimore). marzo de 2017;96(10): 6137.

30. Wang L, Liang S, Chen H, Xu Y, Wang Y. The effects of epidural anaesthesia and analgesia on $\mathrm{T}$ lymphocytes differentiation markers and cytokines in patients after gastric cancer resection. BMC Anesthesiol. 12 de 2019;19(1):102.
31. Kim KH, Kim YH, Yun KY, Han SE, Kim ES, Kwon BS, et al. Laparoscopically assisted suprapubic surgery for adnexal tumors under epidural anesthesia. Minim Invasive Ther Allied Technol. febrero de 2017;26(1):39-43.

32. Składzień T, Andres J, Pasternak A. Measurement of the cerebral saturation for assessment of safety of epidural anaesthesia during abdominal surgery. Folia Med Cracov. 2019;59(4):45-54.

33. Karaca Ö, Pınar HU, Duman E, Doğan R. Laparoscopic gastrostomy under awake thoracic epidural anesthesia: A successful experience. Agri. julio de 2018;30(3):138-41.

34. Jing C, Wang C. Combining Spinal-Epidural Anesthesia versus Single-Shot Spinal Anesthesia for Cesarean Delivery: A Meta-Analysis of 5 Randomized Controlled Trials. Med Sci Monit. 18 de abril de 2019;25:2859-67.

\section{(c) $)$ (i) $(2)$ \\ CREATIVE COMMONS RECONOCIMIENTO-NOCO- MERCIAL-COMPARTIRIGUAL 4.0.}

\section{CitAR ESTE ARTICULO:}

Saquipay Ortega, H. V., \& Bravo Paucar, R. E. (2021). Ventajas del uso de anestesia epidural en cirugía abdominal. RECIMUNDO, 5(1 (Suple), 75-86. https:// doi.org/10.26820/recimundo/5.(Suple1).oct.2021.75-86 